\title{
Optimization of wave pattern of corrugation on plates of heat exchanger
}

\author{
Václav Dvořák ${ }^{1, *}$ \\ ${ }^{1}$ Technical University of Liberec, Faculty of Mechanical Engineering, Department of Power Engineering Equipment. Address: \\ Studentska 2, 46117, Liberec, Czech Republic
}

\begin{abstract}
The article deals with optimization of undulated heat transfer surface of plate heat exchanger. The goal of optimization is not only to increase effectiveness of heat transfer but also to reduce the pressure drop. Using four optimization parameters, the function describing the shape of corrugations is defined and these parameters are optimized. A simple steepest descent gradient method and stochastic Monte Carlo method are used, the optimum shape of the heat exchange surface is searched for four values of the pressure drop. It was found that a simple steepest descent method quickly gets stuck in a local extreme and the resulting configurations were found only by changing the slope of the waves. The stochastic method enables full optimization through the optimization space and find completely different waveforms with similar values of target function. The question, which shape of the waves on the heat transfer surface is the best, is still open.
\end{abstract}

\section{Introduction}

Energy recovery during air ventilation is one of the ways to reduce the energy consumption of buildings. Heat recovery can substantially reduce energy consumption needful for heating during cold seasons or air conditioning during hot seasons. The main component of any energy recovery devices are heat exchangers. Development of recuperative heat exchangers in recent years has focused on increasing their effectiveness. Another challenge is the development of so-called enthalpy exchangers for simultaneous heat and moisture transport, i.e. transport of both sensible and latent heat, as presented by Vít et al. in work [1]. To develop heat exchangers, methods of computational fluid dynamics are increasingly used, but there are still some problems to transfer the calculation results to the design and manufacturing processes.

To simulate a heat exchanger, it is necessary to create a model and a computational mesh and then use a Computational Fluid Dynamics (CFD) software. When assembling the heat exchanger, complicated and irregular narrow channels are created. These channels are split into small volumes (elements). The final meshes are structured or unstructured with different element sizes. Most of researchers use unstructured meshes for their calculations. Gherasim et al., in work [2], presented a comparison of various grids for a plate heat exchanger modeled by a tetrahedral mesh.

Subsequent researchers have used numerical simulations to investigate plate heat exchangers with chevron (undulated) profiles. Tsai [3] and Liu [4], for example, dealt with these heat exchangers and their different geometries. Similarly Giurgiu et al. in work [5] used CFD to study the influence of geometric characteristics of the two plates on the intensification process of heat transfer. They used SST k- $\omega$ turbulence model and non-structural high-density mesh and modeled only small part of the heat exchanger. They carried out also a session of experimental measurements which confirms the results obtained through numerical simulation. Kumar et al. [6] experimentally investigated the effect of symmetric and mixed chevron angles in a plate heat exchanger on heat transfer rate ratio, friction losses and many others quantities.

Rios Iribe et al. [7] investigated, using CFD and hexahedral meshes, momentum and heat transfer of a nonNewtonian fluid in steady laminar regime through a plate heat exchanger at constant wall temperature. Although the flow pattern was highly complex, under an adequate definition of the characteristics parameters, it was possible to establish simple correlations between the dimensionless numbers that characterize the thermalhydraulic behavior of plate heat exchangers. Al-Waked et al. [8] investigated numerically conjugate heat and mass transfer processes across membrane heat exchangers of variable mass transfer resistance. A hybrid mesh was used.

Novosád and Dvořák [9], investigated the influence of oblique waves in herringbone pattern on the heat transfer surface. The main difficulty faced in this work was the creation of custom geometry. Each version had to be modelled, meshed, loaded into the solver, set the boundary conditions and subsequently perform the numerical simulation separately.

Waves in herringbone pattern are widely used for steal plate heat exchanger. The reason is that forming the plates

Corresponding author: vaclav.dvorak@tul.cz 
provides efficient and reliable way to increase the heat transfer area of the heat exchanger. Another pattern is the wavy pattern, visible down in Figure 1, which provides the same advantages. Although it is often used in plastic and aluminium plate heat exchangers in air ventilation systems, it is overlooked by researchers and it is not clear, which of both patterns is better, i.e. has higher heat transfer and lower pressure drop.

It is not even clear whether there is a completely different shape of waves that would be even better. Therefore the aim of this work is to optimize the shape of wavy pattern and answer which of these patter is the ideal one for the most intensive heat transfer and lowest pressure drop.
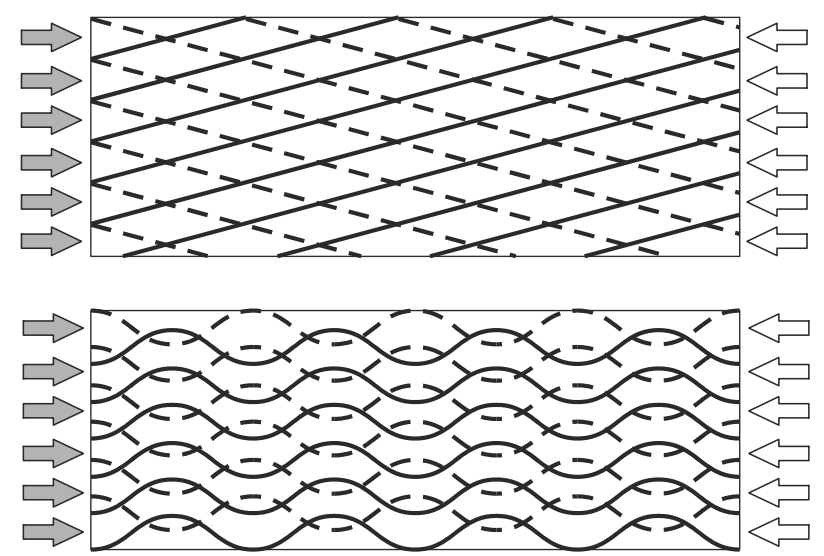

$\Rightarrow$ could air
hot air
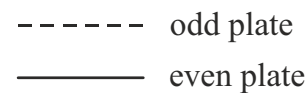

Fig. 1 Two basic types of undulation; up - herringbone pattern, down - wavy pattern with sinusoidal waves.

\section{Methods}

\subsection{Methods of grid generation}

To optimize a plate heat exchanger, we needed to model, mesh and compute a lot of computational variants. However, there are several disadvantages of repeated generation of computational meshes: Demanding and time consuming work and parameterization of the model is problematic. Further, even a small change of geometry requires to do again the whole process of model creation and mesh generation. Furthermore, meshes are not similar, i.e. the size, shape, and height of cells adjacent to walls are not the same for different topologies and numerical error which is added to the solution and influence evaluation of objective function.

Therefore, we used method, developed by Dvořák [10], for generating computational variants. This method is based on a dynamic mesh, which is provided by Ansys Fluent software. The meshes were created by pull procedure, which is similar to the own production process, i.e. the pulling direction is perpendicular to the plates. The main advantage is that this type of mesh generation is automatic and controlled by in-house software. All computational variants thus have the same mesh.
In this paper, we discuss the case of a counter flow heat exchanger, which does not have a symmetrical heat transfer area. It is because undulation cross each other, as it is obvious form Figure 1. To simulate flow and heat transfer in such heat exchanger, we need to create model containing two plates, as it is shown in Figure 2.

The heat transfer surface was divided into two parts. Input and output portions with length of $L_{0}=0.05 \mathrm{~m}$ were fixed, remained flat and served for developing velocity profiles before and after central part.

The central part which was provided by undulation had length $L=0.15 \mathrm{~m}$. Input boundary conditions were specified by velocities of $3 \mathrm{~m} / \mathrm{s}$, the output boundary conditions were specified as pressure outlets with static pressure $0 \mathrm{~Pa}$. Bottom and upper boundary conditions were periodic, thus a heat exchanger with infinite number of plates was simulated. The width of the model was $B=0.05 \mathrm{~m}$. We used mesh density, element size and count of cell layers according to recommendations from Dvořák and Novosád [11].

In this study, we used turbulence model SST k- $\omega$, medium was air considered as incompressible gas. As a results, we obtained pressure, velocity, turbulence fields and temperature fields inside the computational domain for given inlet velocity.

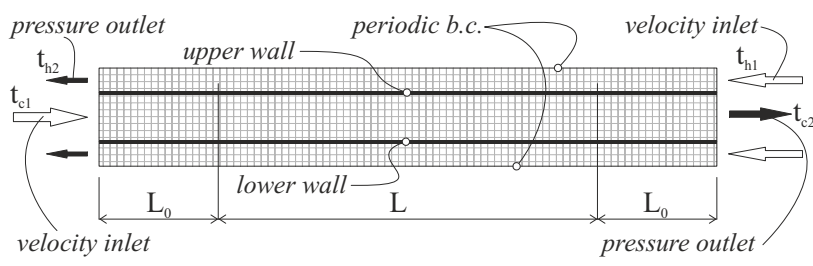

Fig. 2 Model of heat exchange surface of counter flow recuperative heat exchanger.

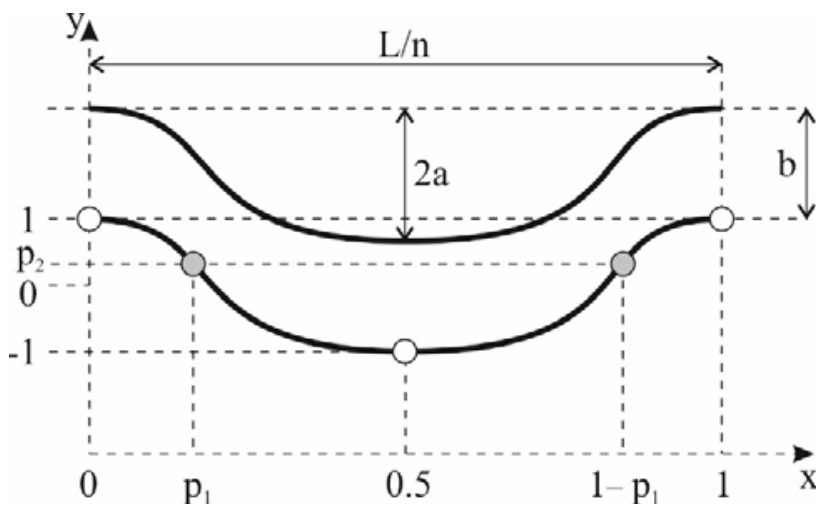

Fig. 3 Definition of parameters defining the wave curve.

In this work, the longitudinal corrugations was formed by the curve in Figure 3. The curve consisted of five points and associated by natural cubic spline. The curve is axially symmetrical. To create a curve, it is necessary to know the length $L(\mathrm{~m})$ of the heat exchanger, number of longitudinal waves $n(1)$, the wave pitch $b(\mathrm{~m})$, their amplitude $a(\mathrm{~m})$ and parameters $\pi_{1}$ and $\pi_{2}$. Parameter $\pi_{1}$ expresses the longitudinal coordinate of the control points, while the parameter $\pi_{2}$ represents vertical position of the two control points. As will be seen later, 
these two parameters allow creation of a wave curve similar to a sine curve and broken wave pattern similar to herringbone pattern.

The shape of the middle part of plates was defined as vertical displacement $z(\mathrm{~m})$ of points on the wall according to equation

$$
z=\frac{p}{2}[\cos (2 \pi m)+s]
$$

where $p=0.003 \mathrm{~m}$ is plate pitch, $s=1$ for upper wall of the model and $s=-1$ for lower wall, $m$ (1) represents wave number which is calculated from horizontal coordinates $(x, y)$ of points on the wall and set of parameters according to relation

$$
m=\frac{1}{b}\left[s y+\frac{a}{2} f\left(2 \pi \frac{n x}{L}\right)+y^{\prime}\right],
$$

where $f(1)$ is function describing wave shape according Figure 3.

Amplitude of wavy corrugation $a(\mathrm{~m})$ was not determined directly, but as multiple of the length of longitudinal waves

$$
a=\pi_{3} \frac{L}{n},
$$

It is, because properties of waves depends on count of waves only slightly, while the dependency on the wave steepness is more significant. Therefore, the steepness of waves, i.e. parameter $\pi_{3}(1)$ become the third optimization parameter. The forth optimization parameter was count of longitudinal waves, i.e. $\pi_{4}=n$.

The method of grid transformation according to relations (1) to (5) are described in work [10].

\subsection{Method for optimization of plate heat exchanger}

To optimize the shape of heat transfer surface of a heat exchanger, we need an objective or target function $F$. Producers of heat exchangers often require as high heat transfer as it is possible for given pressure drop. Therefore and according to previous works [12], we used linear combination of two main characteristic properties of a heat exchanger in the form of

$$
F=\eta-C_{F}\left(\frac{\left|\Delta p-\Delta p_{R}\right|}{\Delta p_{R}}\right)^{R},
$$

where $\Delta p(\mathrm{~Pa})$ is calculated or measured pressure drop, $\Delta p_{R}(\mathrm{~Pa})$ is reference or required pressure loss, $\eta(1)$ is calculated or measured coefficient of effectiveness, $C_{F}$ (1) penalty coefficient and $R(1)$ is parameter by which we can control peak of objective function. Values $C_{F}=5$ and $R=2$ were found in previous work [13]. Thus, obtained effectiveness is penalized by the difference between obtained and required pressure drop. Suggested function has quite flat peak which simplifies searching for an optimum.

The pressure drop was defined as pressure difference

$$
\Delta p=\bar{p}_{01}-\bar{p}_{2}
$$

where $\bar{p}_{01}(\mathrm{~Pa})$ is mass-averaged total pressure at the inlet and $\bar{p}_{2}(\mathrm{~Pa})$ is area-averaged static pressure at the pressure outlet.

The coefficient of effectiveness (or effectiveness) is the efficiency of sensible heat transfer. Most recuperative heat exchangers in air conditioning systems work in isobaric mode, where mass flow rates of hot and cold air are equal, i.e. $\dot{m}_{h}=\dot{m}_{c}$. Assuming parity between specific heat capacities $c_{p h}=c_{p c}$, we can write the effectiveness as

$$
\eta=\frac{t_{h 1}-t_{h 2}}{t_{h 1}-t_{c 1}},
$$

where $t_{h 1}\left({ }^{\circ} \mathrm{C}\right)$ and $t_{h 2}\left({ }^{\circ} \mathrm{C}\right)$ are inlet and outlet temperatures of hot air, $t_{c 1}\left({ }^{\circ} \mathrm{C}\right)$ is outlet temperature of cold air.

The task of optimization dealt with finding of optimal values of four parameters according to Figure 3; $\mathrm{x}$ coordinates $\pi_{1}$ and y-coordinates $\pi_{2}$ of control points on the wave, steepness of waves $\pi_{3}$ and number of longitudinal waves $\pi_{4}=n$, while plate pitch $p$, often defined by requirement on material consumption, and pitch of undulation $b$, which is usually defined by deformability of plate material and technology of pressing, remained constant during optimization.

As optimization method, we first used common steepest descent gradient optimization method [14] which belongs to so-called local method, because these method are capable to find only a local optimum. Since it was found during research that the problem has a lot of optimums, a global optimization method was applied.

As global optimization method, we used a simple stochastic Monte Carlo algorithm described in work [15]. To emphasize searching for local optimum, we used modification described in previous work [14] that favoured variants closer to the last best variant found according to relations

$$
\begin{aligned}
& \pi_{i}^{n}=\pi_{i}^{\text {best }}-\operatorname{ran}^{2}\left(\pi_{i}^{\text {best }}-\pi_{i}^{\text {low }}\right), \\
& \pi_{i}^{n}=\pi_{i}^{\text {best }}+\operatorname{ran}^{2}\left(\pi_{i}^{u p}-\pi_{i}^{\text {best }}\right),
\end{aligned}
$$

where $\pi_{i}^{n}$ is a new value of optimization parameter $\pi_{i}$, $\pi_{i}^{\text {best }}$ is the value of the parameter for the last best variant, $\pi_{i}^{\text {low }}$ and $\pi_{i}^{u p}$ are lower and upper limit of the same parameter respectively, and $\operatorname{ran}=\langle 0 ; 1\rangle$ is random number. The use of equations (7) and (8) were alternated also randomly.

The model of plate heat exchanger was optimized for four values of reference pressure, $\Delta p_{R}=100,150,200$ and $250 \mathrm{~Pa}$. 


\section{Results}

\subsection{Steepest descent method}

First the optimization was performed using a simple gradient method, which is able to find only a local optimum of the objective function. The optimization was initialized by values of parameters:, $\pi_{1}=0.3, \pi_{2}=0.5$ and $\pi_{3}=0.08$, while $\pi_{4}=5$. In this optimization, the parameter $\pi_{4}$ remained constant.

The results in the figures show that the steepest descent gradient optimization method is very fast and relatively quickly leads to the goal. The method relatively quickly finds a local minimum.

Table 1. Results of optimization no. 1, steepest descent method, $\pi_{4}=5$, and four values of reference pressure $\Delta p_{R}$.

\begin{tabular}{|l|c|c|c|c|}
\hline$\Delta p_{R}(\mathrm{~Pa})$ & 100 & 150 & 200 & 250 \\
\hline No. of steps & 19 & 72 & 76 & 78 \\
\hline$\eta(1)$ & 0.7816 & 0.8079 & 0.822 & 0.8335 \\
\hline$\Delta p(\mathrm{~Pa})$ & 102.3 & 150.95 & 200.72 & 250.62 \\
\hline$F(1)$ & 0.7789 & 0.8047 & 0.822 & 0.8335 \\
\hline$\pi_{1}(1)$ & 0.3 & 0.3329 & 0.3414 & 0.3464 \\
\hline$\pi_{2}(1)$ & 0.504 & 0.4942 & 0.4944 & 0.4931 \\
\hline$\pi_{3}=a \cdot n / L(1)$ & 0.0798 & 0.1972 & 0.227 & 0.2454 \\
\hline$\pi_{4}=n(1)$ & 5 & 5 & 5 & 5 \\
\hline
\end{tabular}

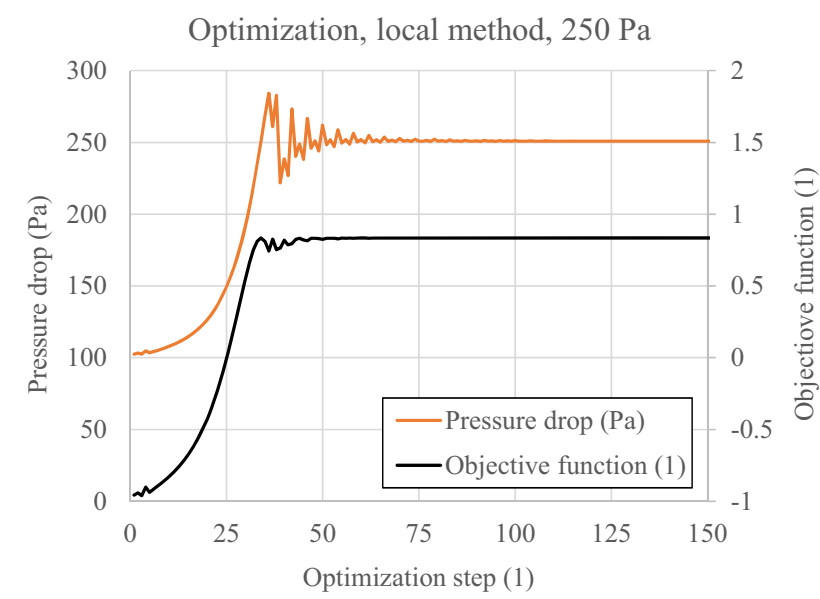

Fig. 4 Course of optimization for reference pressure $\Delta p_{R}=250 \mathrm{~Pa}$, while steepest descent method is used.

Optimization results are summarized in Table 1, examples of the course of the objective function and the diagrams are in Figures 4 and 5. As can be seen in the course of the objective function $F$, and the pressure drop $\Delta p$ in Figure 4 , the search for wave configuration was very fast, the desired pressure is achieved in practice after 34 steps.

However, this method consists in finding the gradient direction in which the target function is changing most rapidly. It turned out that the objective function is most sensitive on parameter $\pi_{3}$, i.e. on the slope of the waves. Therefore, during the optimization, the optimum value of parameter $\pi_{3}$ was found soonest, while other parameters remained practically unchanged, see diagram in Figure 5.

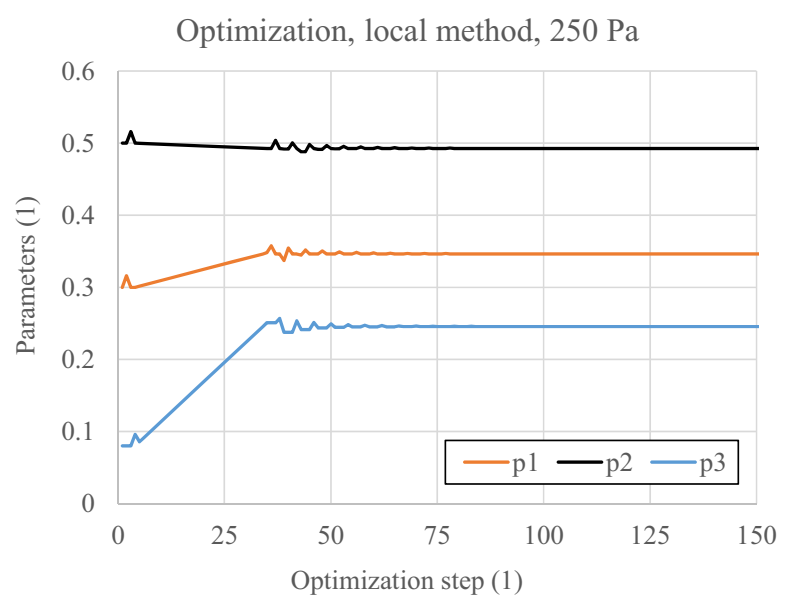

Fig. 5 Development of parameters during optimization for reference pressure $\Delta p_{R}=250 \mathrm{~Pa}$ by steepest descent method.

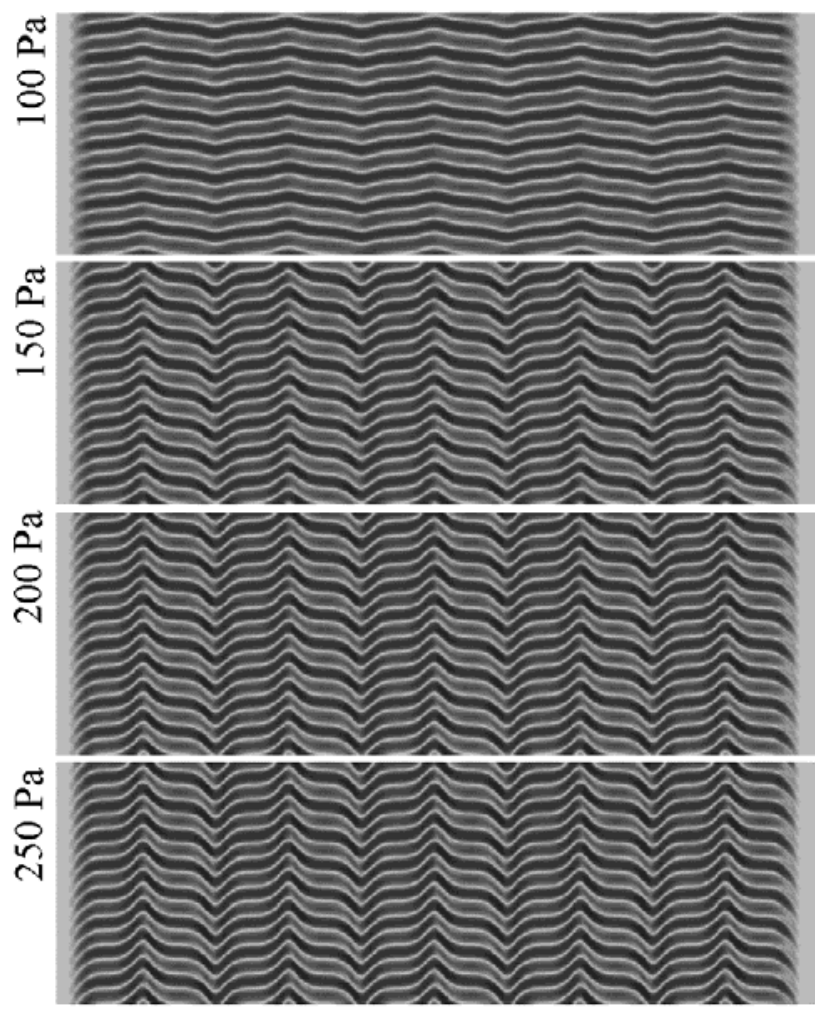

Fig. 6 Optimized shapes of heat transfer surfaces for various values of reference pressure, steepest descent method is used.

As it is apparent from the results of optimization and especially Figure 6, the result of optimization was largely influenced by the initial values of parameters. Because the value of the objective function is strongly dependent on the amplitude of the waves, the third parameter $\pi_{3}$ was the most developed. In contrast, the parameter $\pi_{2}$ changes only slightly and parameter $\pi_{1}$ only negligibly. This situation did not change in the further optimization, which should take place already in the other direction. Also found shapes of waves indicates that only a local optimum was found. We can observe in Figure 6 that 
found shapes differ only in the third parameter, i.e. steepness of the waves. It is therefore obvious that optimization method got stuck in local extreme and we need to use a method capable to identify a global maximum.

\subsection{Stochastic Monte Carlo global method}

The results of applying stochastic Monte Carlo method are summarized in Table 2. From this it follows that the optimization takes incomparably longer, but the value of the objective function do not different too much from the values that can be found in Table 1 for much simpler and quicker gradient method.

Table 2. Results of optimization no. 2 for stochastic method, four values of reference pressure $\Delta p_{R}$.

\begin{tabular}{|l|c|c|c|c|}
\hline$\Delta p_{R}(\mathrm{~Pa})$ & 100 & 150 & 200 & 250 \\
\hline No. of steps & 312 & 217 & 411 & 712 \\
\hline$\eta(1)$ & 0.7814 & 0.8088 & 0.8295 & 0.8414 \\
\hline$\Delta p(\mathrm{~Pa})$ & 101.07 & 150.55 & 199.41 & 252.29 \\
\hline$F(1)$ & 0.7809 & 0.8087 & 0.8295 & 0.8410 \\
\hline$\pi_{1}(1)$ & 0.4408 & 0.2034 & 0.1895 & 0.4484 \\
\hline$\pi_{2}(1)$ & 0.5487 & 0.2439 & 0.6622 & 0.9397 \\
\hline$\pi_{3}=a \cdot n / L(1)$ & 0.0293 & 0.2046 & 0.2022 & 0.294 \\
\hline$\pi_{4}=n(1)$ & 2 & 8 & 4 & 6 \\
\hline
\end{tabular}

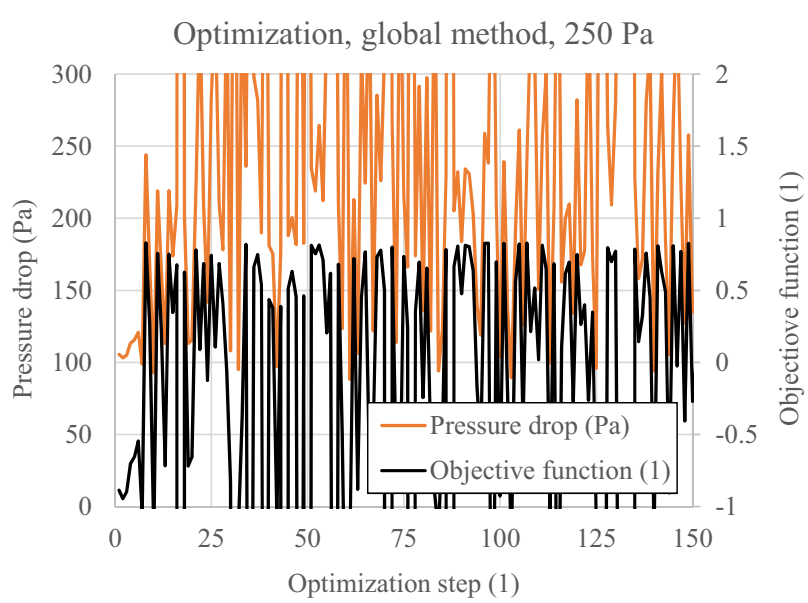

Fig. 7 Course of optimization for reference pressure $\Delta p_{R}=250 \mathrm{~Pa}$, while stochastic method is used.

The advantage of used stochastic method is that it does not get stuck in a local extreme, but it can leave it. As can be seen from Figure 7, the method is able to find relatively quickly a configuration of heat transfer area with the required pressure drop, moreover, it is able to explore other options too.

Yet, according to Figure 8, it appears that even this method has a tendency to remain in the first found optimum, i.e. with the height of the target function found it is less likely that other configurations of heat exchange surface is explored. As we can see on the development of the optimization parameters in Figure 8, optimization parameters cease to evolve in time.

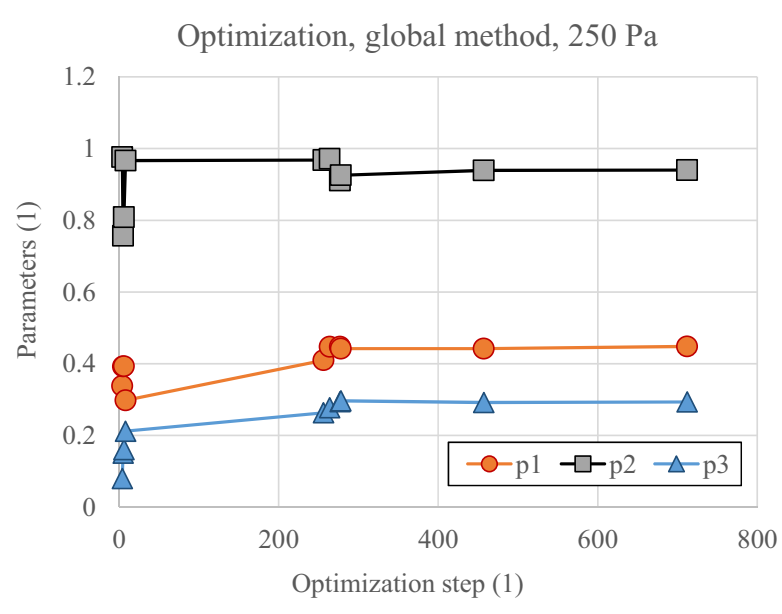

Fig. 8 Development of parameters during optimization for stochastic method and reference pressure $\Delta p_{R}=250 \mathrm{~Pa}$.

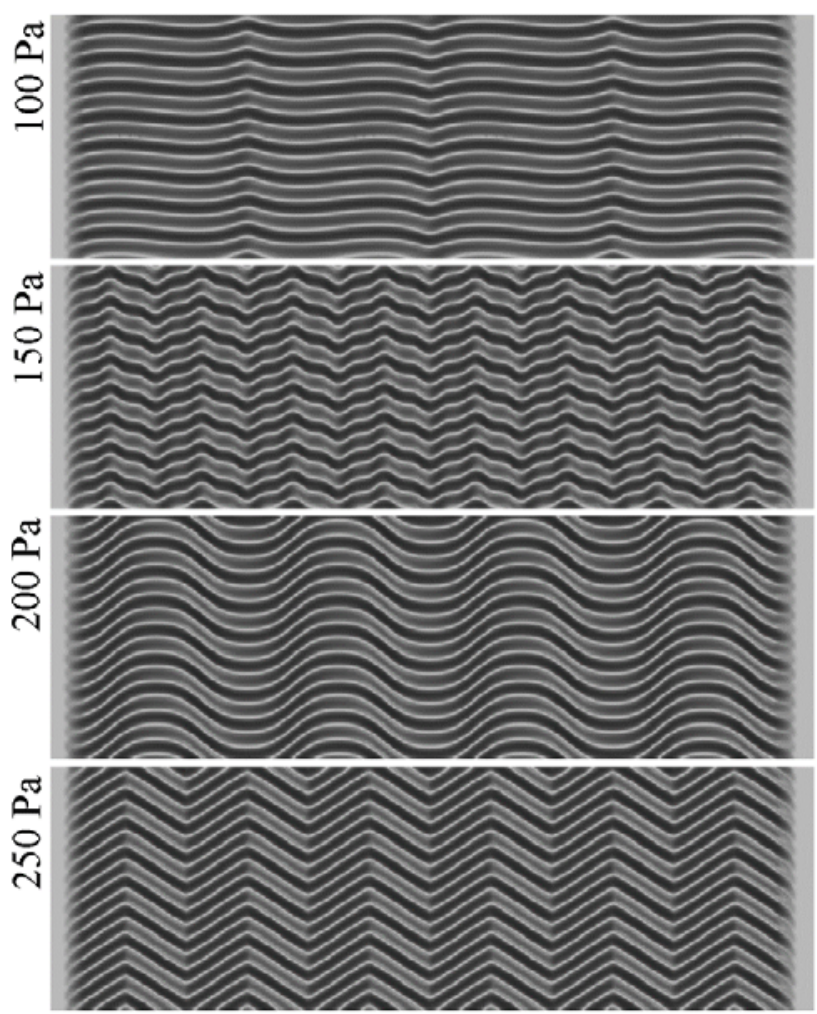

Fig. 9 Optimized shapes of heat transfer surfaces for various values of reference pressure, stochastic method is used.

As seen from the results of the individual and particularly from Figure 9, in which the resulting shapes are shown, stochastic method can find advantageous shapes in virtually any point of the optimization space. The result can thus be for example very long waves $\left(\pi_{4}=2\right.$ for $\left.\Delta p_{R}=100 \mathrm{~Pa}\right)$ or very short and corrugated $\left(\Delta p_{R}=150\right.$ $\mathrm{Pa})$ or rounded smooth waves $\left(\Delta p_{R}=200 \mathrm{~Pa}\right)$ or even variation of the herringbone pattern $\left(\Delta p_{R}=250 \mathrm{~Pa}\right)$. It follows that the optimization method works and allows to find the variation of the heat exchange surface. However, the question whether the global optimum was found in not answered. Also, we still do not know whether the waves are better rounded, or shaped in herringbone patter or in other design. 
Interesting is certainly the recognition that totally different configurations can reach very similar results.

Since this run of optimization did not reply asked questions, one more optimization was conducted, in which the values of only three parameters were sought, while the number of waves remained the same, i.e. $\pi_{4}=5$.

Table 3. Results of optimization no. 3, stochastic method is used, $\pi_{4}=5$, four values of reference pressure $\Delta p_{R}$.

\begin{tabular}{|l|c|c|c|c|}
\hline$\Delta p_{R}(\mathrm{~Pa})$ & 100 & 150 & 200 & 250 \\
\hline No. of steps & 179 & 217 & 176 & 190 \\
\hline$\eta(1)$ & 0.7798 & 0.8073 & 0.8333 & 0.8378 \\
\hline$\Delta p(\mathrm{~Pa})$ & 100.85 & 151.96 & 203.74 & 250.57 \\
\hline$F(1)$ & 0.7794 & 0.8065 & 0.8316 & 0.8378 \\
\hline$\pi_{1}(1)$ & 0.2433 & 0.2561 & 0.1972 & 0.4439 \\
\hline$\pi_{2}(1)$ & 0.3807 & 0.3834 & 0.4089 & 0.9264 \\
\hline$\pi_{3}=a \cdot n / L(1)$ & 0.0668 & 0.2024 & 0.2446 & 0.308 \\
\hline$\pi_{4}=n(1)$ & 5 & 5 & 5 & 5 \\
\hline
\end{tabular}

As can be seen in Table 3, the resulting value of the objective function is not very different from the values found for the four parameters. Found shapes this time are much more similar and have the shape of a fish bone, see Figure 10.

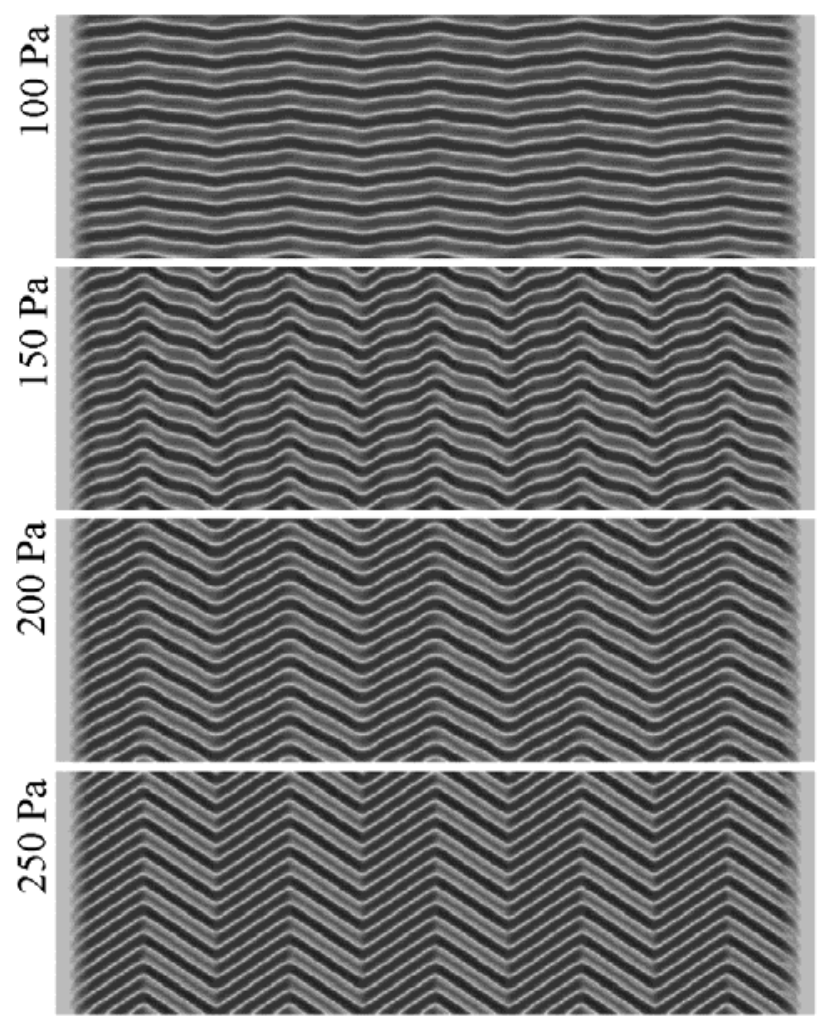

Fig. 10 Optimized shapes of heat transfer surfaces for various values of reference pressure, stochastic method is used, three parameters are optimized.

From the course of the development of optimization parameters in Figure 11, it seems that when it finds a high value of the objective function, there is only a slight shift values of optimization parameters.

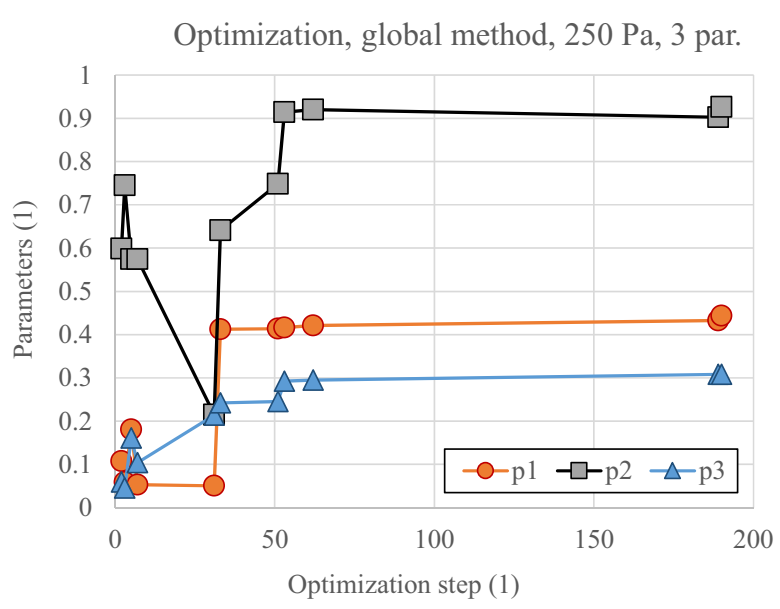

Fig. 11 Development of parameters during optimization for stochastic method and reference pressure $\Delta p_{R}=250 \mathrm{~Pa}$.

\section{Conclusions}

An undulated heat transfer surface of recuperative heat exchanger was optimized to obtain as high coefficient of effectiveness of heat transfer as possible. The shape of waves of corrugation for surface of heat exchanger was optimized.

The task of optimization was to find optimal values of four parameters of wavy pattern: x-coordinate and $y$ coordinate of control points on wave curve, steepness of waves and count of longitudinal waves.

The optimization procedure looked for maximum of objective function which was a linear combination of effectiveness and pressure drop. First we used simple steepest descent method and then stochastic Monte Carlo method and the optimum was searched for four values of reference pressure drop. Three different optimization were run and we investigated the resulting shapes of undulation.

It was found that simple steepest descent method is fast, but only a local minimum is found and it is reached simply by altering steepness of waves.

The stochastic Monte Carlo method is more capable to search through the optimization space. Very different shapes were found as optimal - sharp waves, rounded, or even waves in herringbone pattern. It was verified that a global optimization method is necessary to use, preferably in conjunction with local method.

In further work, it would be also beneficial to carried out an analysis of flow around optimal shapes.

\section{Acknowledgment}

This publication was written at the Technical University of Liberec, Faculty of Mechanical Engineering with the support of the Institutional Endowment for the Long Term Conceptual Development of Research Institutes, as provided by the Ministry of Education, Youth and Sports of the Czech Republic in the year 2017. 


\section{References}

1. T. Vít, P. Novotný, V.V. Nguyen, V. Dvořák, WSEAS, Recent Advances in Energy, Environment, Economics and Technological Innovation, 34-38 (2013)

2. I. Gherasim, N. Galanis, C.T. Nguyen, Int. Jour. of Thermal Science. 50, 1499-1511 (2011)

3. Y.Ch. Tsai, F.B. Liu, P.T. Shen, Int. Commun. in Heat and Mass Transfer 36, 574-578 (2009)

4. F.B. Liu, Y.Ch. Tsai, Heat Mass Transf. 46, 585-593 (2010)

5. O. Giurgiu, A. Pleşa, L. Socaciu. Energy Procedia 85, 244-251 (2016)

6. V. Kumar, A.K. Tiwari, S.K. Ghosh. Energy Conversion and Manag. 118, 142-154 (2016)

7. E.Y. Rios-Iribe, M.E. Cervantes-Gaxiola, E. RubioCastro, O.M. Hernández-Calderón, Applied Thermal Eng.

8. R. AL-Waked, M.S. Nasif, G. Morrison, M. Behnia, Applied Thermal Eng. 84, 301-309 (2015)

9. J. Novosád, V. Dvořák, EPJ Web of Conferences 67, 02085 (2014)

10. V. Dvořák, Recent Advances in Computer Science 1, 193-198 (2014)

11. V. Dvořák, J. Novosád, Recent Advances in Computer Science, pp 115-120 (2015)

12. V. Dvořák, Applied Mechanics \& Materials 752-753, 820-827 (2015)

13. V. Dvoř́a, MATEC Web of Conferences 76, 04027 (2016)

14. R. Fletcher M. J. D. Powell, A Rapidly Convergent Descent Method for Minimization. Computer J. 6 (2): 163-168 (1963).

15. Metropolis P., Ulam S.: The Monte-Carlo method. J. of the American Statistical Assoc. 44, 335, (1949) 